\title{
Left ventricular rotational abnormalities following successful kidney transplantation-insights from the three-dimensional speckle-tracking echocardiographic MAGYAR-Path Study
}

\author{
Bernadett Borda ${ }^{1}$, Árpád Kormányos ${ }^{2}$, Péter Domsik², Anita Kalapos², Csaba Lengyel ${ }^{2}$, Nóra Ambrus², \\ György Lázár ${ }^{1}$, Tamás Forster ${ }^{2}$, Attila Nemes ${ }^{2}$ \\ ${ }^{1}$ Department of Surgery, ${ }^{2} 2$ nd Department of Medicine and Cardiology Centre, ${ }^{3} 1$ st Department of Medicine, Faculty of Medicine, Albert Szent- \\ Györgyi Clinical Center, University of Szeged, Szeged, Hungary \\ Correspondence to: Attila Nemes, MD, PhD, DSc, FESC. 2nd Department of Medicine and Cardiology Centre, Faculty of Medicine, Albert Szent- \\ Györgyi Clinical Centre, University of Szeged, Semmelweis street 8, P.O. Box 427, H-6725 Szeged, Hungary. Email: nemes@in2nd.szote.u-szeged.hu.
}

Background: Kidney transplantation (KTx) is the preferred treatment for virtually all suitable candidates with end-stage renal disease. There is limited information on left ventricular (LV) rotational mechanics post-KTx, therefore the present study aimed to assess it and to compare it to that of age- and gendermatched healthy controls.

Methods: The present study comprised $42 \mathrm{KTx}$ patients, from which 4 patients were excluded due to insufficient image quality (mean age: $46.3 \pm 8.2$ years, 29 males). Control group consisted of 81 age- and gender-matched healthy individuals (mean age: $43.5 \pm 10.8$ years, 51 males). All KTx patients and healthy controls successfully underwent transthoracic two-dimensional (2D) Doppler echocardiography and three-dimensional speckle-tracking echocardiography (3DSTE).

Results: Significant differences could be demonstrated in LA diameter, LV end-diastolic diameter and volume, interventricular septum, LV posterior wall thickness, LV ejection fraction and early and late filling transmitral flow velocities and in their ratio between KTx patients and controls; none of the subjects examined showed grade 2-4 mitral and/or tricuspid regurgitations. Three patients following successful KTx showed near absence of LV twist called as LV 'rigid body rotation' (RBR) movement. When the remaining 35 post-KTx patients were analysed separately, reduced basal LV rotation could be demonstrated in postKTx patients with tendentious increase in apical LV rotation resulting in an unchanged LV twist.

Conclusions: KTx is associated with alterations in LV rotational mechanics with unchanged $\mathrm{LV}$ twist suggesting a remodelling of this sort of movement. The near absence of LV twist (LV-RBR) could be demonstrated in some post-KTx cases.

Keywords: Kidney transplantation (KTx); left ventricular rotation (LV rotation); twist; three-dimensional (3D); echocardiography

Submitted Sep 19, 2018. Accepted for publication Oct 26, 2018.

doi: $10.21037 /$ qims.2018.10.12

View this article at: http://dx.doi.org/10.21037/qims.2018.10.12

\section{Introduction}

Kidney transplantation (KTx) is the preferred treatment for virtually all suitable candidates with end-stage renal disease (ESRD) (1). Compared to dialysis, KTx improves both survival and quality of the life of the patients. In patients following successful KTx, leading causes of death are cardiovascular diseases (CVDs) (1). Theoretically, early detection of CVD-related alterations in myocardial mechanics could help in the selection of high-risk patients. Echocardiography is a widely used method of choice in 
assessing different cardiac functions (2). Three-dimensional (3D) speckle-tracking echocardiography (3DSTE) has the ability to perform non-invasive $3 \mathrm{D}$ evaluation of cardiac mechanics including left ventricular (LV) volumetric, strain and rotational analysis at the same time from the same digitally stored dataset (3). The net difference between $\mathrm{LV}$ apical and basal rotations called as LV twist is considered to be a significant part of LV function $(4,5)$. 3DSTE has been validated for quantitative assessment of LV twist (3). There is limited information on $\mathrm{LV}$ rotational mechanics post-KTx, therefore the present study aimed to assess it and to compare it to that of age- and gender-matched healthy controls.

\section{Methods}

\section{Patient population}

The present study comprised $42 \mathrm{KTx}$ patients, from which 4 patients were excluded due to insufficient image quality (mean age: $46.3 \pm 8.2$ years, 29 males). All patients were sent from the KTx Unit at the Department of Surgery, University of Szeged minimum one year after the operation. The control group consisted of 81 age- and gender-matched healthy individuals (mean age: $43.5 \pm 10.8$ years, 51 males). None of the healthy subjects and KTx patients had chest pain, other cardiovascular symptoms or previous myocardial infarction. None of KTx patients received antirejection therapy. All KTx patients and healthy controls successfully underwent transthoracic two-dimensional (2D) Doppler echocardiography extended with 3DSTE at the same time. The institution's human research committee approved the study, which also complied with the ethical guidelines set by the 1975 Declaration of Helsinki. All patients gave informed consent. The present study is part of the MAGYAR-Path Study (Motion Analysis of the heart and Great vessels bY three-dimensionAl speckle-tRacking echocardiography in Pathological cases), which aimed to examine pathophysiological consequences of different pathological states on myocardial mechanics among others ('magyar' means 'Hungarian' in Hungarian language).

\section{D Doppler echocardiography}

Experienced operators (Péter Domsik, Anita Kalapos) carried out routine transthoracic measurements including quantification of LV dimensions, volumes and ejection fraction and left atrial (LA) dimensions. Toshiba Artida ${ }^{\mathrm{TM}}$ echocardiography system (Toshiba Medical Systems, Tokyo, Japan) with a PST-30SBP (1-5 MHz) phasedarray transducer was used for measurements. Valvular regurgitations were visually assessed, valvular stenoses were excluded by colour Doppler echocardiography, while mitral inflow E/A was measured by pulsed Doppler.

\section{DSTE}

The same Toshiba Artida ${ }^{\mathrm{TM}}$ echocardiographic machine (Toshiba Medical Systems, Tokyo, Japan) was used for 3DSTE assessments. 3DSTE was performed following recent practices using apical window for data acquisitions (3). With a PST-25SX matrix-array transducer, six wedgeshaped subvolumes were acquired during a single breathhold and constant RR interval by the same experienced operators with sector width as narrow as possible to reach optimal resolution images. Acquired datasets were forwarded for offline image analysis using 3D Wall Motion Tracking software version 2.7 (Toshiba Medical Systems, Tokyo, Japan). From the 3D datasets, apical two- (AP2CH) and four-chamber $(\mathrm{AP} 4 \mathrm{CH})$ views and 3 short-axis views at end-diastole were chosen automatically by the software. Following optimization of imaging planes and definition of mitral annular end-points and LV apex, 3D endocardial surface was automatically reconstructed and tracked in 3D space throughout the cardiac cycle. Curves were generated by the software for quantification of the following $\mathrm{LV}$ rotational and twist parameters:

* LV basal rotation = degree of clockwise rotation of the LV basal myocardial segments;

* LV apical rotation = degree of counterclockwise rotation of the LV apical myocardial segments;

* LV twist = net difference between LV basal and apical rotations;

* Time-to-peak LV twist from the start of the heart cycle.

In some cases, $\mathrm{LV}$ basal and apical rotations were in the same direction called as LV 'rigid body rotation' (RBR) (6). In these subjects, proper LV twist data could not be gathered, therefore apicobasal gradient defined as endsystolic LV apical minus LV basal rotation was calculated (Figure 1).

\section{Statistical analysis}

All data are reported as mean \pm standard deviation. Statistical significance was considered if $\mathrm{P}<0.05$. Categorical 

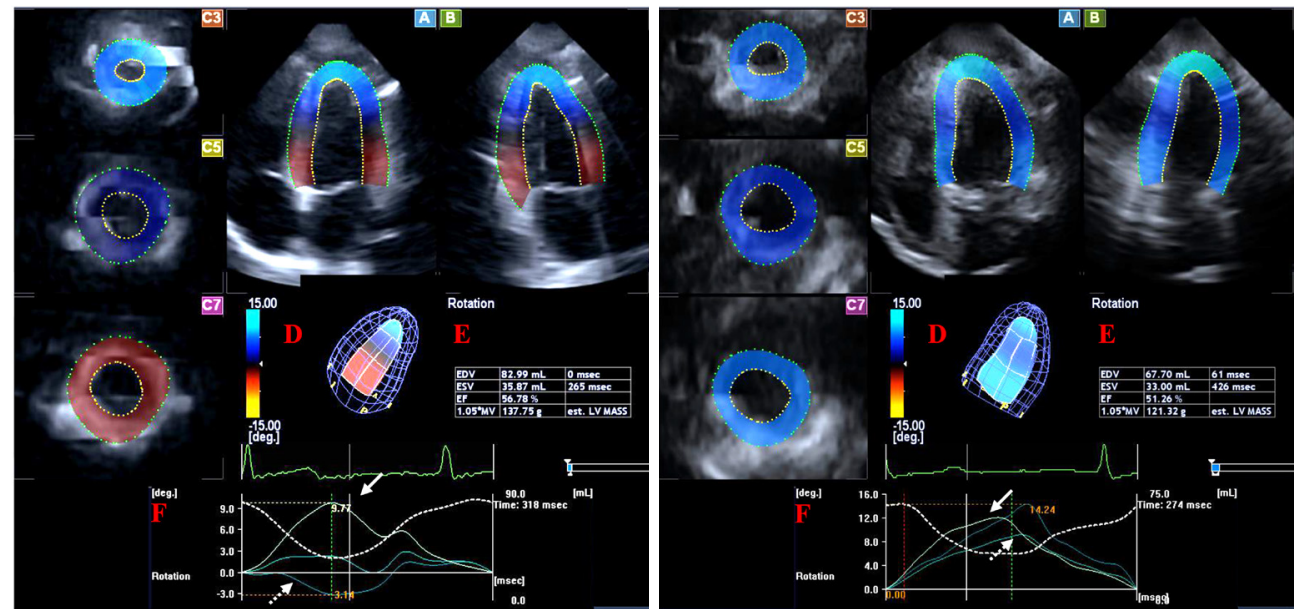

Figure 1 A healthy subject with normal left ventricular (LV) rotation (left panel), and a patient following successful kidney transplantation (KTx) with LV 'rigid body rotation' (RBR) (right panel) are presented. Apical 4-chamber (A) and 2-chamber (B) views and short-axis views (C3, C5, C7) at different LV levels could be seen. The 3D mesh model of the left ventricle (D) and calculated LV volumetric (E) and rotational (F) data are also presented. Dashed line represents LV volume changes during the cardiac cycle, while white line represents LV rotational parameters. $\mathrm{LV}$ basal rotation is clockwise directed (dashed arrow), while LV apical rotation is counter-clockwise directed (white arrow) in the healthy subject (left panel). Both LV apical and basal rotations are counterclockwise directed suggesting LV-RBR in the KTx patient (right panel).

variables were assessed by Fisher's exact test. ShapiroWilks test was used to test normal distribution in every dataset. Levene's test for equality of variances was used for assessing homogeneity of variance. Student's $t$-test was used for assessing datasets in normal distribution and Mann-Whitney-Wilcoxon test in datasets that were not normally distributed. Statistical analysis was carried out by using RStudio \{RStudio Team [2015]. RStudio: Integrated Development for R. RStudio, Inc., Boston, MA, USA\}. Commercially available software package was used for offline data analysis and graph creation (MATLAB 8.6, The MathWorks Inc., Natick, MA, USA. 2015).

\section{Results}

\section{Demographic, clinical and routine $2 D$ echocardiographic data}

The ratio of risk factors and most important medications applied in post-KTx patients are listed in Table 1. Significant differences could be demonstrated in LA diameter, LV enddiastolic diameter and volume, interventricular septum, $\mathrm{LV}$ posterior wall thickness, $\mathrm{LV}$ ejection fraction and early and late filling transmitral flow velocities and in their ratio between post-KTx patients and controls (Table 1). None of the control subjects and post-KTx patients showed valvular stenosis or grade 2-4 mitral and/or tricuspid regurgitations.

\section{DSTE-derived LV rotation and twist}

Three patients following successful KTx showed near absence of LV twist called as LV-RBR movement. Due to this special movement, their results were managed separately. When the remaining 35 post-KTx patients were analysed separately, reduced basal $L V$ rotation could be demonstrated in post-KTx patients with tendentious increase in apical $\mathrm{LV}$ rotation resulting in an unchanged $\mathrm{LV}$ twist (Table 2).

\section{$L V-R B R$}

Three patients with LV-RBR showed abnormally directed counterclockwise basal LV rotation $(5.13 \pm 3.29$ degrees) and similar but normally directed counterclockwise apical rotation $(14.33 \pm 10.72$ degrees). Therefore, real LV twist could not be measured only LV apicobasal gradient considered to be as their net difference $(9.20 \pm 9.04$ degrees $)$ (Figure 1).

\section{Discussion}

CVDs are the leading causes of morbidity and mortality in chronic kidney disease patients. KTx is the treatment modality of choice for virtually all suitable candidates with ESRD $(7,8)$. The leading causes of death in patients who 
Table 1 Baseline demographic and two-dimensional echocardiographic data of patients following successful kidney transplantation and of healthy controls

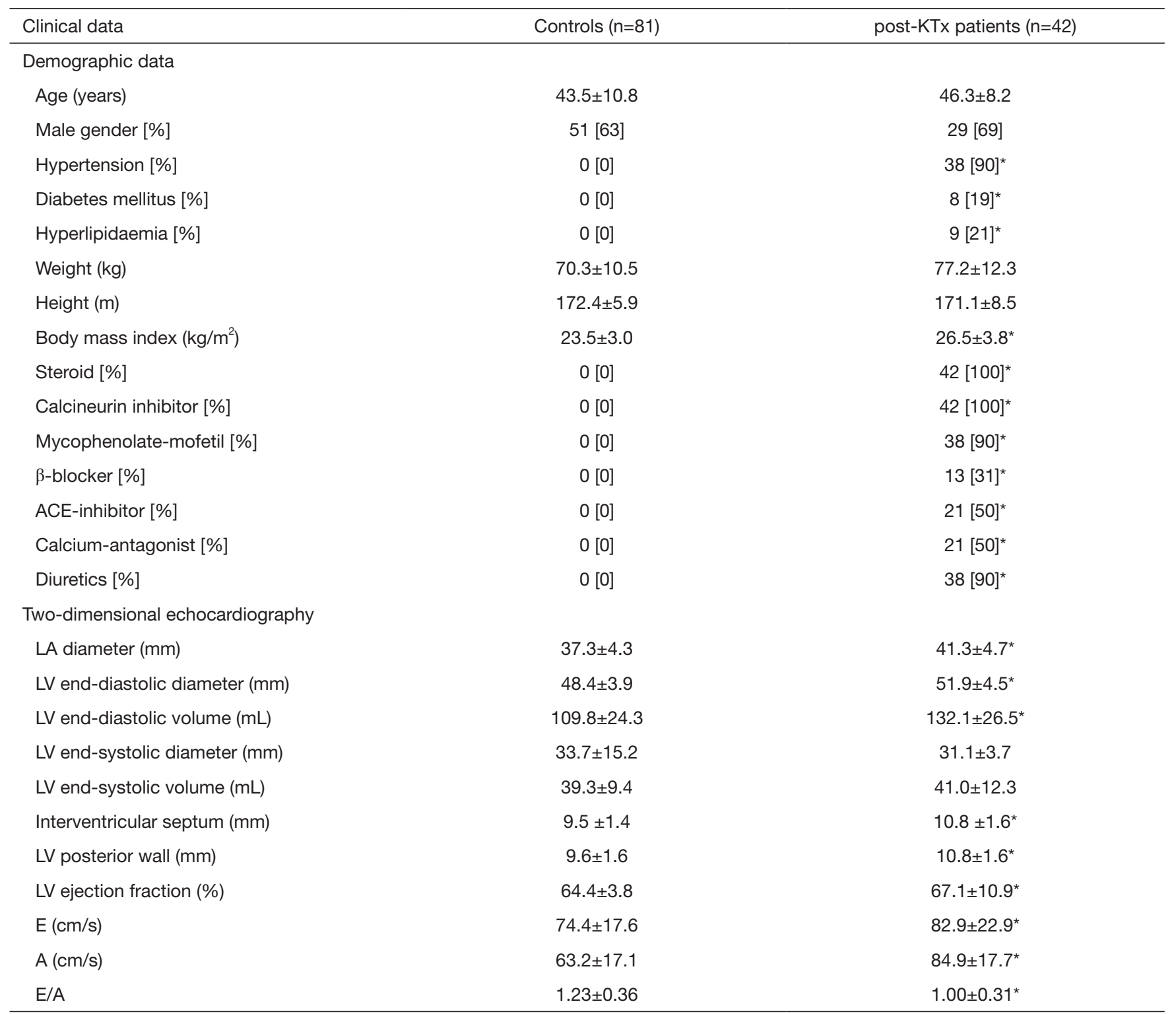

*, $\mathrm{P}<0.05$ vs. controls. ACE, angiotensin-converting enzyme; KTx, kidney transplantation; LA, left atrial; LV, left ventricular; E and A, early and late diastolic transmitral flow velocity.

Table 2 Three-dimensional speckle-tracking echocardiography-derived left ventricular volumetric and rotational parameters in patients following successful kidney transplantation and in healthy controls

\begin{tabular}{lcc}
\hline LV rotational parameters & Controls $(\mathrm{n}=81)$ & Post-KTx patients without LV-RBR $(\mathrm{n}=35)$ \\
\hline Basal LV rotation (degree) & $-4.22 \pm 2.14$ & $-3.34 \pm 1.81^{*}$ \\
Apical LV rotation (degree) & $9.68 \pm 4.00$ & $10.55 \pm 3.67$ \\
LV twist (degree) & $13.90 \pm 4.33$ & $13.89 \pm 3.72$ \\
Time of peak LV twist (ms) & $329 \pm 83$ & $312 \pm 48$ \\
\hline
\end{tabular}

\footnotetext{
*, $\mathrm{P}<0.05$ vs. controls. KTx, kidney transplantation; LV, left ventricular; LV-RBR, left ventricular 'rigid body rotation'.
} 
died with a functioning allograft are CVDs as well (9), which account for almost 40 percent of all deaths in this population. Studies suggest that the survival advantage of KTx may be largely attributed to the reduction in CVDs. In a retrospective analysis of the United States Renal Data System, data of 60,141 adult primary KTx recipients transplanted between 1995 and 2000 were compared with that of 66,813 adult patients on the waiting list over the same time period, Meier-Kriesche et al. have demonstrated progressive decrease in cardiovascular death rates by renal transplant vintage for both diabetic and non-diabetic recipients of both living and deceased donor transplants (10).

Diastolic dysfunction is frequently observed in ESRD, moreover, heart failure (HF) with preserved LV-EF is more common in hemodialysis patients than HF with low LV-EF (11). Hassanin et al. demonstrated that in chronic kidney disease, although longitudinal and radial systolic functions were reduced, LV-EF may remain within normal limits due to the preservation of the circumferential functions (12). KTx was found to significantly improve LV$\mathrm{EF}$ and apical 4-chamber strain and to reduce LV mass index and relative wall thickness. Other variables including global longitudinal strain and diastolic dysfunction were not found to be improved significantly (13).

In a healthy subject, there are special LV rotational mechanics including smaller clockwise LV basal rotation and larger counterclockwise LV apical rotation resulting in $L V$ twist due to two orthogonally directed $L V$ muscle bands (4). LV twist has an essential role in maintaining LV pump function (5). However, its clinical importance is not well established in the general population, and in KTx patients either. 3DSTE-derived LV rotation and twist parameters have already been validated (14). It is known that $\mathrm{LV}$ twisting mechanism is affected by several conditions and disorders, but the results are conflicting (15-17). To this point, however, there is limited information on the relationship between CKD, KTx and LV rotational mechanics. According to Yildirim et al. LV rotational and twist parameters did not differ between those of predialysis and transplantation patient groups (18). In contrast, Deng et al. found that LV basal and apical rotations, average twist and torsion were higher following successful KTx as compared to pre-KTx results (19). Interestingly there was no significant difference between the absolute value of $\mathrm{LV}$ basal and apical rotations in this study.

The present study demonstrated reduced LV basal rotation and slight increase in $\mathrm{LV}$ apical rotation resulting in unchanged LV twist in KTx patients as compared to matched healthy controls. These results suggest a sort of remodelling of $\mathrm{LV}$ rotational mechanics in post-KTx patients. Moreover, significant LV rotational abnormalities (LV-RBR) could also be confirmed in some KTx cases due to abnormally directed $\mathrm{LV}$ basal rotation. Recently these sorts of LV rotational abnormalities could be detected in high ratio of patients with noncompaction cardiomyopathy $(55-100 \%)(20,21)$, cardiac amyloidosis $(60 \%)(22)$, acromegaly (25\%) (23), but reports showed similar cases with other diseases as well. Significant apicobasal gradient (overrotation of the LV apex) suggesting a compensating mechanism could also be detected in these cases similarly to what happens during stress (24). In post-KTx patients, ESRD-related consequences could explain our findings, but the effect of surgical treatment could not be excluded either (18). Further studies are warranted to confirm our findings and to compare them to renal and other functional parameters.

\section{Limitation section}

Several important limitations arose during the assessments which are listed below:

* Comparing pre- and post-transplantation data would have been the most optimal to see whether KTx has any effect on LV rotational mechanics. Unfortunately, it was not possible to make this sort of measurements and comparisons due to technical reasons. Further studies are warranted to compare pre- and post-KTx LV rotational parameters with those of matched control subjects whether KTx has any short- and long-term additive value on them.

* Other LV deformation parameters including LV strains were not assessed in this study. Moreover, 3DSTE-derived different strains of the left and right atria and volumetric data of heart chambers were not analysed between the groups.

* 3DSTE has low temporal and spatial resolutions which could theoretically affect the results.

* Coronary angiography was not performed in any of the KTx patients to exclude coronary artery disease. However, none of them had any clinical signs of angina pectoris or had previous myocardial infarction or other signs of vascular diseases.

* The effect of corresponding risk factors on the results could not be excluded.

* The present study did not aim to validate LV rotational parameters. 


\section{Conclusions}

KTx is associated with alterations in LV rotational mechanics with unchanged LV twist suggesting a remodelling of this sort of movement. The near absence of $\mathrm{LV}$ twist (RBR of the LV) could be demonstrated in some post-KTx cases.

\section{Acknowledgements}

None.

\section{Footnote}

Conflicts of Interest: The authors have no conflicts of interest to declare.

Ethical Statement: The institution's human research committee approved the study (ethical ID: 71/2011), which also complied with the ethical guidelines set by the 1975 Declaration of Helsinki. Written informed consent was obtained from all patients for publication of this manuscript and any accompanying images.

\section{References}

1. Rao NN, Coates PT. Cardiovascular Disease After Kidney Transplant. Semin Nephrol 2018;38:291-7.

2. Nemes A, Forster T. Recent echocardiographic examination of the left ventricle - from M-mode to 3D speckle-tracking imaging. Orv Hetil 2015;156:1723-40.

3. Nemes A, Kalapos A, Domsik P, Forster T. Threedimensional speckle-tracking echocardiography -- a further step in non-invasive three-dimensional cardiac imaging. Orv Hetil 2012;153:1570-7.

4. Nemes A, Kalapos A, Domsik P, Forster T. Left ventricular rotation and twist of the heart. Clarification of some concepts. Orv Hetil 2012;153:1547-51.

5. Nakatani S. Left ventricular rotation and twist: why should we learn? J Cardiovasc Ultrasound 2011;19:1-6.

6. Nemes A, Kormányos Á, Domsik P, Kalapos A, Lengyel C, Valkusz Z, Forster T. Left ventricular 'rigid body rotation' in a patient with acromegaly (from the MAGYAR-Path Study). Quant Imaging Med Surg 2017;7:378-9.

7. Kasiske BL, Chakkera HA, Roel J. Explained and unexplained ischemic heart disease risk after renal transplantation. J Am Soc Nephrol 2000;11:1735-43.

8. Sarnak MJ, Levey AS, Schoolwerth AC, Coresh J, Culleton
B, Hamm LL, McCullough PA, Kasiske BL, Kelepouris E, Klag MJ, Parfrey P, Pfeffer M, Raij L, Spinosa DJ, Wilson PW; American Heart Association Councils on Kidney in Cardiovascular Disease, High Blood Pressure Research, Clinical Cardiology, and Epidemiology and Prevention. Kidney disease as a risk factor for development of cardiovascular disease: a statement from the American Heart Association Councils on Kidney in Cardiovascular Disease, High Blood Pressure Research, Clinical Cardiology, and Epidemiology and Prevention. Circulation 2003;108:2154-69.

9. Ojo AO. Cardiovascular complications after renal transplantation and their prevention. Transplantation 2006;82:603-11.

10. Meier-Kriesche HU, Schold JD, Srinivas TR, Reed A, Kaplan B. Kidney transplantation halts cardiovascular disease progression in patients with end-stage renal disease. Am J Transplant 2004;4:1662-8.

11. Ogawa T, Koeda M, Nitta K. Left Ventricular Diastolic Dysfunction in End-Stage Kidney Disease: Pathogenesis, Diagnosis, and Treatment. Ther Apher Dial 2015;19:427-35.

12. Hassanin N, Alkemary A. Early Detection of Subclinical Uremic Cardiomyopathy Using Two-Dimensional Speckle Tracking Echocardiography. Echocardiography 2016;33:527-36.

13. Hamidi S, Kojuri J, Attar A, Roozbeh J, Moaref A, Nikoo $\mathrm{MH}$. The effect of kidney transplantation on speckled tracking echocardiography findings in patients on hemodialysis. J Cardiovasc Thorac Res 2018;10:90-4.

14. Zhou Z, Ashraf M, Hu D, Dai X, Xu Y, Kenny B, Cameron B, Nguyen T, Xiong L, Sahn DJ. Threedimensional speckle-tracking imaging for left ventricular rotation measurement: an in vitro validation study. $\mathrm{J}$ Ultrasound Med 2010;29:903-9.

15. Phillips AA, Cote AT, Bredin SS, Warburton DE. Heart disease and left ventricular rotation - a systematic review and quantitative summary. BMC Cardiovasc Disord 2012;12:46.

16. Nemes A, Dézsi L, Domsik P, Kalapos A, Forster T, Vécsei L. Left ventricular deformation abnormalities in a patient with calpainopathy-a case from the three-dimensional speckle-tracking echocardiographic MAGYAR-Path Study. Quant Imaging Med Surg 2017;7:685-90.

17. Nemes A, Katona M, Domsik P, Kalapos A, Forster T. Different patterns of left ventricular "rigid body rotation" in 8-year-old twins with anamnestic twin-to-twin transfusion syndrome (from the MAGYAR-Twin Study). 
Quant Imaging Med Surg 2017;7:140-1.

18. Yildirim U, Gulel O, Eksi A, Dilek M, Demircan S, Sahin $M$. The effect of different treatment strategies on left ventricular myocardial deformation parameters in patients with chronic renal failure. Int J Cardiovasc Imaging 2018;34:1731-9.

19. Deng Y, Pandit A, Heilman RL, Chakkera HA, Mazur MJ, Mookadam F. Left ventricular torsion changes post kidney transplantation. J Cardiovasc Ultrasound 2013;21:171-6.

20. Peters F, Khandheria BK, Libhaber E, Maharaj N, Dos Santos C, Matioda H, Essop MR. Left ventricular twist in left ventricular noncompaction. Eur Heart J Cardiovasc Imaging 2014;15:48-55.

21. Kalapos A, Domsik P, Forster T, Nemes A. Comparative evaluation of left ventricular function by two-dimensional echocardiography and three-dimensional speckle-tracking echocardiography in noncompaction cardiomyopathy. Results from the MAGYAR-Path Study. Orv Hetil

Cite this article as: Borda B, Kormányos Á, Domsik P, Kalapos A, Lengyel C, Ambrus N, Lázár G, Forster T, Nemes A. Left ventricular rotational abnormalities following successful kidney transplantation-insights from the three-dimensional speckletracking echocardiographic MAGYAR-Path Study. Quant Imaging Med Surg 2018;8(11):1095-1101. doi: 10.21037/ qims.2018.10.12
2013;154:1352-9.

22. Nemes A, Földeák D, Domsik P, Kalapos A, Sepp R, Borbényi Z, Forster T. Different patterns of left ventricular rotational mechanics in cardiac amyloidosisresults from the three-dimensional speckle-tracking echocardiographic MAGYAR-Path Study. Quant Imaging Med Surg 2015;5:853-7.

23. Kormányos Á, Domsik P, Kalapos A, Orosz A, Lengyel C, Valkusz Z, Trencsányi A, Forster T, Nemes A. Left ventricular twist is impaired in acromegaly: Insights from the three-dimensional speckle tracking echocardiographic MAGYAR-Path Study. J Clin Ultrasound 2018;46:122-8.

24. Nemes A, Szántó G, Domsik P, Kormányos Á, Kalapos A, Ambrus N, Forster T. Change of left ventricular "rigid body rotation" during dipyridamole-induced vasodilation: A case from the three-dimensional speckle tracking echocardiographic MAGYAR-Stress Study. J Clin Ultrasound 2018;46:152-6. 